\title{
BMJ Open Age-standardised incidence rate and epidemiology of colorectal cancer in Africa: a systematic review and meta- analysis
}

\author{
Nina Arhin, ${ }^{1}$ Paddy Ssentongo (1) , ${ }^{2}$ Morris Taylor, ${ }^{2}$ Elizabeth Josephine Olecki, ${ }^{3}$ \\ Colette Pameijer (D) , ${ }^{3}$ Chan Shen, ${ }^{3}$ John Oh, ${ }^{3}$ Cathy Eng ${ }^{1}$
}

To cite: Arhin N, Ssentongo P,

Taylor M, et al. Agestandardised incidence rate and epidemiology of colorectal cancer in Africa: a systematic review and meta-analysis. BMJ Open 2022;12:e052376. doi:10.1136/ bmjopen-2021-052376

- Prepublication history and additional supplemental material for this paper are available online. To view these files, please visit the journal online (http://dx.doi.org/10.1136/ bmjopen-2021-052376).

NA and PS contributed equally.

NA and PS are joint first authors.

Received 13 April 2021

Accepted 29 November 2021

Deck for updates

(c) Author(s) (or their employer(s)) 2022. Re-use permitted under CC BY-NC. No commercial re-use. See rights and permissions. Published by BMJ.

For numbered affiliations see end of article.

Correspondence to Dr Paddy Ssentongo; pssentongo@pennstatehealth. psu.edu

\section{ABSTRACT}

Objectives Colorectal cancer (CRC) is the second-leading cause of cancer deaths globally, with low-income and middle-income countries (LMICs) disproportionately affected. Estimates of CRC rates in LMIC are scarce. We aimed to (1) estimate sex-specific incidence of CRC, (2) estimate temporal trend and (3) determine regional variations of $\mathrm{CRC}$ rates on the African continent.

Design Systematic review and meta-analysis

Methods PubMed (MEDLINE), OVID (MEDLINE), Scopus and Cochrane Library databases were systematically searched from inception to 12 December 2020. We included populationbased studies that reported the incidence or prevalence estimates of CRC in Africa. Studies not conducted in humans or did not directly report the rates of CRC were excluded. Random effects model was used to pool the estimates. The methodological quality of studies was assessed with the Newcastle-Ottawa Scale.

Outcome measures Overall and sex-specific annual agestandardised incidence rates (ASIR) of CRC per 100000 population.

Results The meta-analysis included 14 studies consisting of 3365 individuals with CRC (mean age, 58 years, $53 \%$ male). The overall ASIR of CRC in Africa per 100000 population was 5.25 (95\% Cl 4.08 to 6.75). The rates were slightly higher in males (4.76) than in females (4.18), but not significantly different. Subgroup analysis indicated greater point estimates in North Africa (8.66) compared with sub-Saharan Africa (5.91); and higher estimates in Eastern (8.29) and Northern (8.66) Africa compared with Western (3.55) and Southern (3.57) Africa, but not statistically significant. The overall trend in ASIR has remained constant at nearly 5 per 100000 population for the last 6 decades.

Conclusion CRC estimates in Africa are heterogeneous and could be underestimated. High-quality data collection systems such as population-based cancer registries may facilitate accurate estimation of country-specific rates and provide critical information which would be lucrative to the consideration of resources needed for screening, early detection, treatment and improving overall patient outcomes.

\section{INTRODUCTION}

Colorectal cancer (CRC) is the third most common cancer and the second-leading cause of cancer deaths globally. ${ }^{1}$ In 2018 , there were
Strengths and limitations of this study

- All United Nations (UN) regions (North Africa and sub-Saharan Africa) were represented.

- UN subregions (Eastern, Western, Southern and Northern) of Africa were represented.

- Country-level data came from only $18 \%$ of the continent.

- Middle Africa had no data on colorectal cancer prevalence.

an estimated 1.8 million new cases of CRC diagnoses and 862000 deaths from CRC. ${ }^{1}$ The majority of deaths from cancer occurred in low-income and middle-income countries (LMICs), with most patients presenting with late-stage disease and commonly unable to obtain medical treatment services.

Even though the incidence of CRC has always been considered to be lower in LMIC, compared with high-income countries, the rates of CRC have been increasing in LMIC over time. ${ }^{2}$ The rising incidence of cancer in LMIC has been attributed in part to the adoption of high-risk lifestyles such as smoking, excessive alcohol use, physical inactivity, as well as an ageing population. ${ }^{3}$

Africa is the second largest and second most populated continent with an estimated population of 1.3 billion people in 2018, accounting for $16 \%$ of the world's human population. ${ }^{4}$ Despite this vast population, CRC in Africa is not currently well characterised, in part due to deficiencies in the data on the incidence, prevalence and mortality of all cancers in Africa. A vast majority of available data come from existing, limited cancer registries which cover less than half of the population. ${ }^{5}$ Nevertheless, based on current and available data, CRC is considered the fifth most common cancer in Africa. ${ }^{6}$ The rate of CRC is estimated to be higher in Northern 

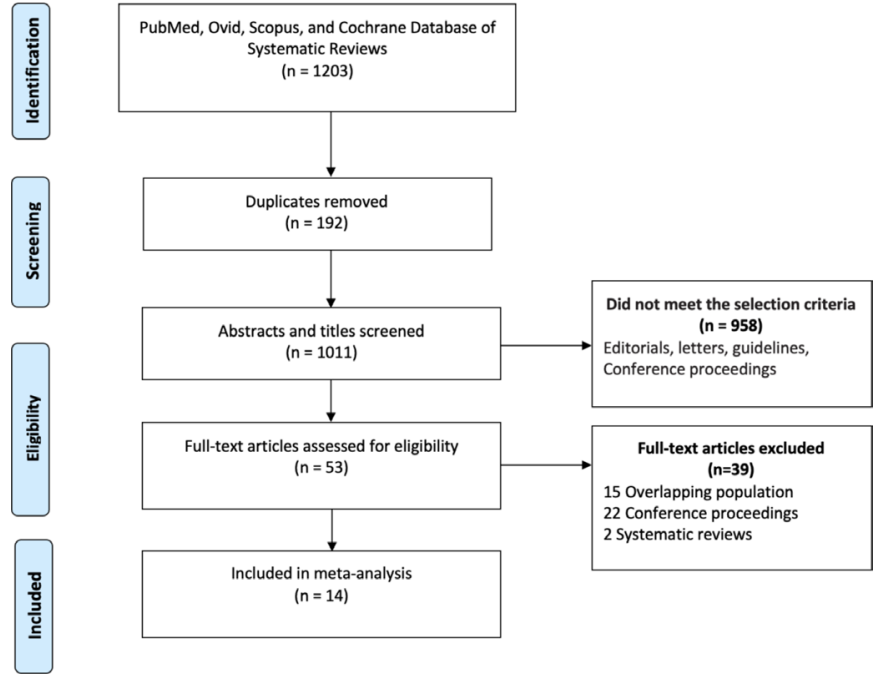

ㅉّㅁ

Figure 1 PRISMA flow chart of a systematic review of colorectal cancer incidence in Africa. PRISMA, Preferred Reporting Items for Systematic Reviews and Meta-Analyses.

Africa than sub-Saharan Africa (SSA) due to the absence of screening systems and population-based cancer registries (PBCR) in SSA. ${ }^{56}$

This systematic review and meta-analysis aims to comprehensively characterise and estimate the incidence of CRC based on available data. These estimates will raise awareness regarding the current incidence of CRC in Africa and will guide future public health allocation of resources to prevent, control and treat CRC.

\section{METHODS}

\section{Search strategy and selection criteria}

This study adheres to the reporting guidance provided in the Preferred Reporting Items for Systematic Reviews and Meta-Analyses in online supplemental table $1 .{ }^{78} \mathrm{We}$ searched PubMed (MEDLINE), OVID (MEDLINE), Scopus and Cochrane Library databases from inception to 12 December 2020 for articles reporting the incidence rates of CRCs in Africa. We searched the grey or difficult to locate literature, including Google Scholar and preprint servers. We performed handsearching of the reference lists of included studies, relevant reviews or other relevant documents. The search terms of interest were identified by using Medical Search Headings (MeSH). They included "colorectal cancer" OR "colon cancer" OR "rectal cancer" OR" colorectal carcinoma” AND "epidemiology" OR "incidence" OR "prevalence" AND " Africa”. Duplicate studies were initially extracted via Endnote software. Three reviewers (NA, MT and PS) independently screened titles and abstracts of the studies for inclusion eligibility. The comprehensive list of studies found from the initial search was transferred into Endnote, which further removed duplicate studies. The inclusion criteria for this meta-analysis and systematic review were defined as studies that (1) reported the incidence or prevalence estimates of CRC in Africa; (2) were conducted in human subjects and (3) were population based (all cases

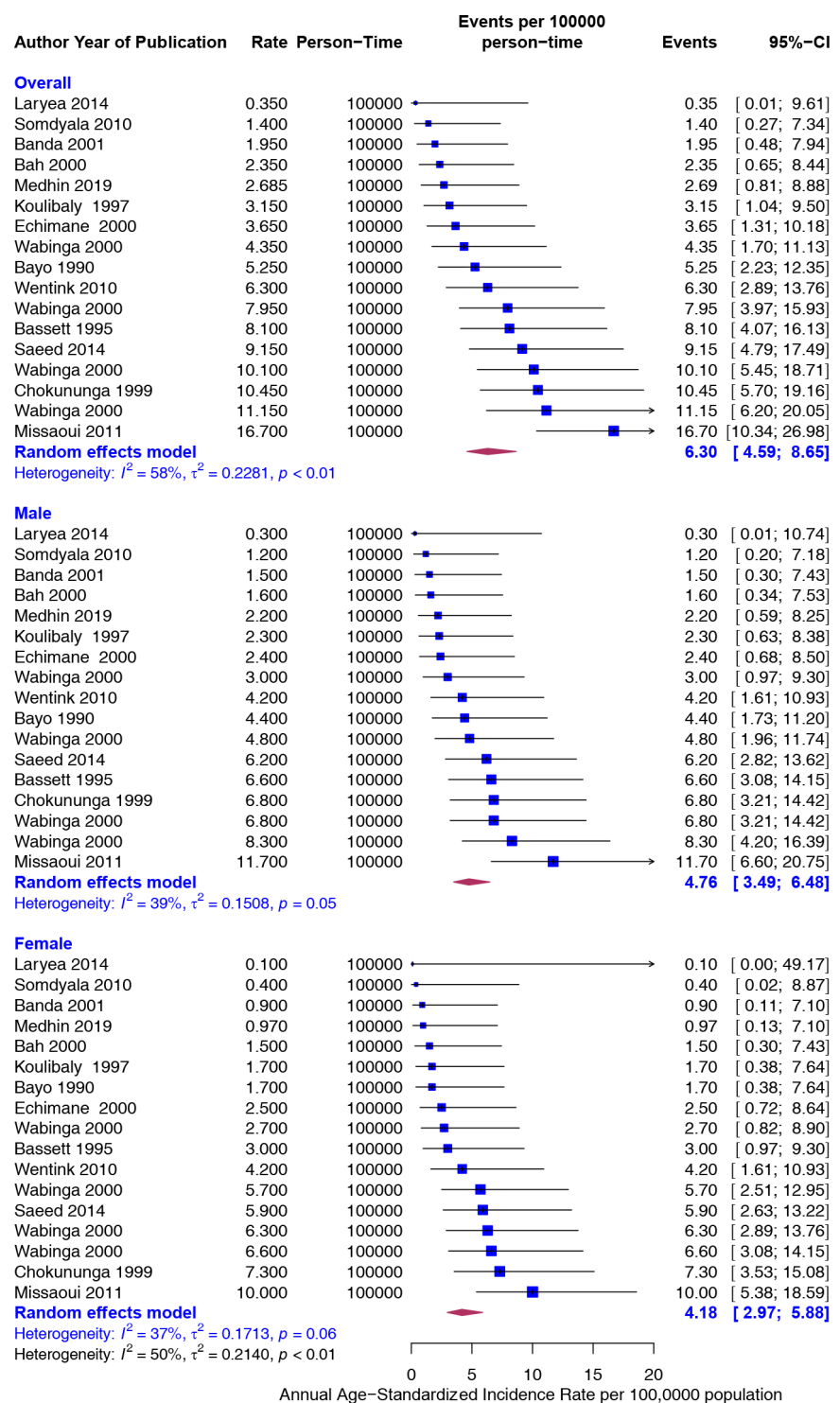

Figure 2 Overall and sex-specific annualised agestandardised incidence rates (ASIR) of colorectal cancer in Africa. Event values represent the ASIR of colorectal cancer per 100000 population. Blue squares and their corresponding lines are the point estimates and $95 \% \mathrm{Cls}$. Maroon diamonds represent the pooled estimate of the ASIR, overall and by sex (width denotes $95 \% \mathrm{Cl}$ ). Although not statistically significant, the pooled ASIR of colorectal cancer in Africa was higher in males $\left(I^{2}=39\right)$ than in females $\left(I^{2}=37\right)$. P for interaction comparing the different subgroups $=0.37$.

in a defined geographical area, or hospital or communitybased surveillance). Excluded studies were not conducted in humans or did not directly report the rates of CRC, metaanalyses, literature reviews or commentaries.

\section{Patient and public involvement}

No patient and public involvement in this systematic review and meta-analysis.

\section{Data extraction and quality assessment}

After the reviewers initially screened titles and abstracts of potential articles, full-text articles were independently 


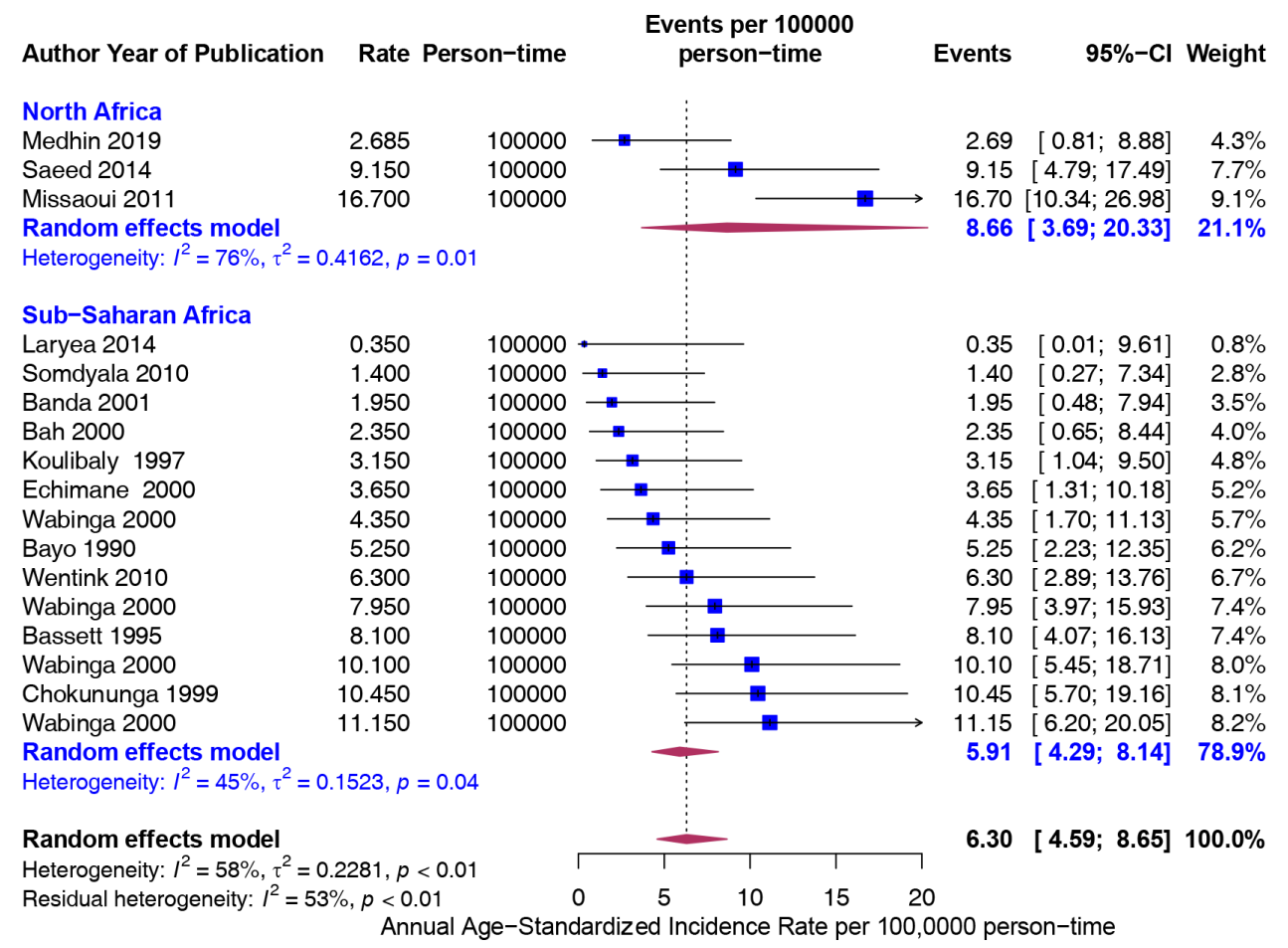

Figure 3 Overall age-standardised incidence rates (ASIR) (per 100000 population) of colorectal cancer in Africa stratified by United Nations region (North Africa and sub-Saharan Africa). Event values represent the ASIR of colorectal cancer per 100000 population. Blue squares and their corresponding lines are the point estimates and $95 \% \mathrm{Cls}$. Maroon diamonds represent the pooled estimate of the ASIR, overall and by United Nations regions (width denotes 95\% CI). Although not statistically significant, the pooled ASIR of colorectal cancer in Africa was higher in North Africa $\left(I^{2}=76\right)$ than SSA $\left(I^{2}=45\right)$. $P$ for interaction comparing the different subgroups=0.21. SSA, sub-Saharan Africa.

screened by three reviewers (NA, MT and PS) for eligibility. In the event of a discrepancy regarding an article's inclusion, a consensus was reached by discussion. Articles that met inclusion criteria had appropriate data extracted using a standard data collection form. We extracted the following information: the year of publication, country, region, cohort and cohort year, study design, sample size, gender percent, sample size of patients with CRC. If duplicate articles identified, we included only mutually exclusive data.

\section{Assessment of methodological quality of the papers}

The methodological quality of studies was assessed with the Newcastle-Ottawa Scale, a validated tool for assessing quantitative cross-sectional, case-control and cohort studies. ${ }^{9}$ Scores of 8 to the maximum score of 9 were defined as high quality, scores of $5-7$ as intermediate quality, and scores of 1-4 as low quality.

\section{Standardisation}

Age standardisation of incidence rates was carried out by the direct method, using age specific rates for 5-year age groups and the world standard population and was reported by each paper. ${ }^{10}$

\section{Statistical analysis}

The primary outcome of interest was the overall and sexspecific annual age-standardised incidence rate (ASIR) of CRC . The metaprop function from the $\mathrm{R}$ package meta was used to calculate the pooled effect estimates using random effects models. ${ }^{11}$ We applied the DerSimonian and Laird random effects method to estimate the pooled between-study variance (heterogeneity). ${ }^{12}$ Individual and pooled estimates were graphically displayed using forest plots. A random effects model assumes the observed estimates of CRC can vary across studies because of real differences in the effect in each study as well as sampling variability (chance). Between-study heterogeneity was assessed using $\mathrm{I}^{2}$ statistics, expressed as \% (low $(25 \%)$, moderate $(50 \%)$ and high $(75 \%)$ and Cochrane's $Q$ statistic (significance level $<0.05){ }^{13}$ To investigate the sources of heterogeneity, we conducted subgroup analyses using year of study (Before and after the year 2000), United Nations regions (SSA vs North Africa) and United Nations subregions (Western, Eastern, Southern, Northern and Eastern Africa). Results were reported as the annual ASIR per 100000 person-time. Potential ascertainment bias (as might be caused by publication bias) was assessed with funnel plots by plotting the study effect size against SEs of the effect size and Egger/Begg test. ${ }^{14}$ All statistical analyses were performed with $\mathrm{R}$ software, V.4.0.3 (R Core Team, Vienna, Austria).

\section{RESULTS}

\section{Study selection}

Our initial searches yielded 1203 studies, of which 53 underwent full-text screening (figure 1). Of these, 22 


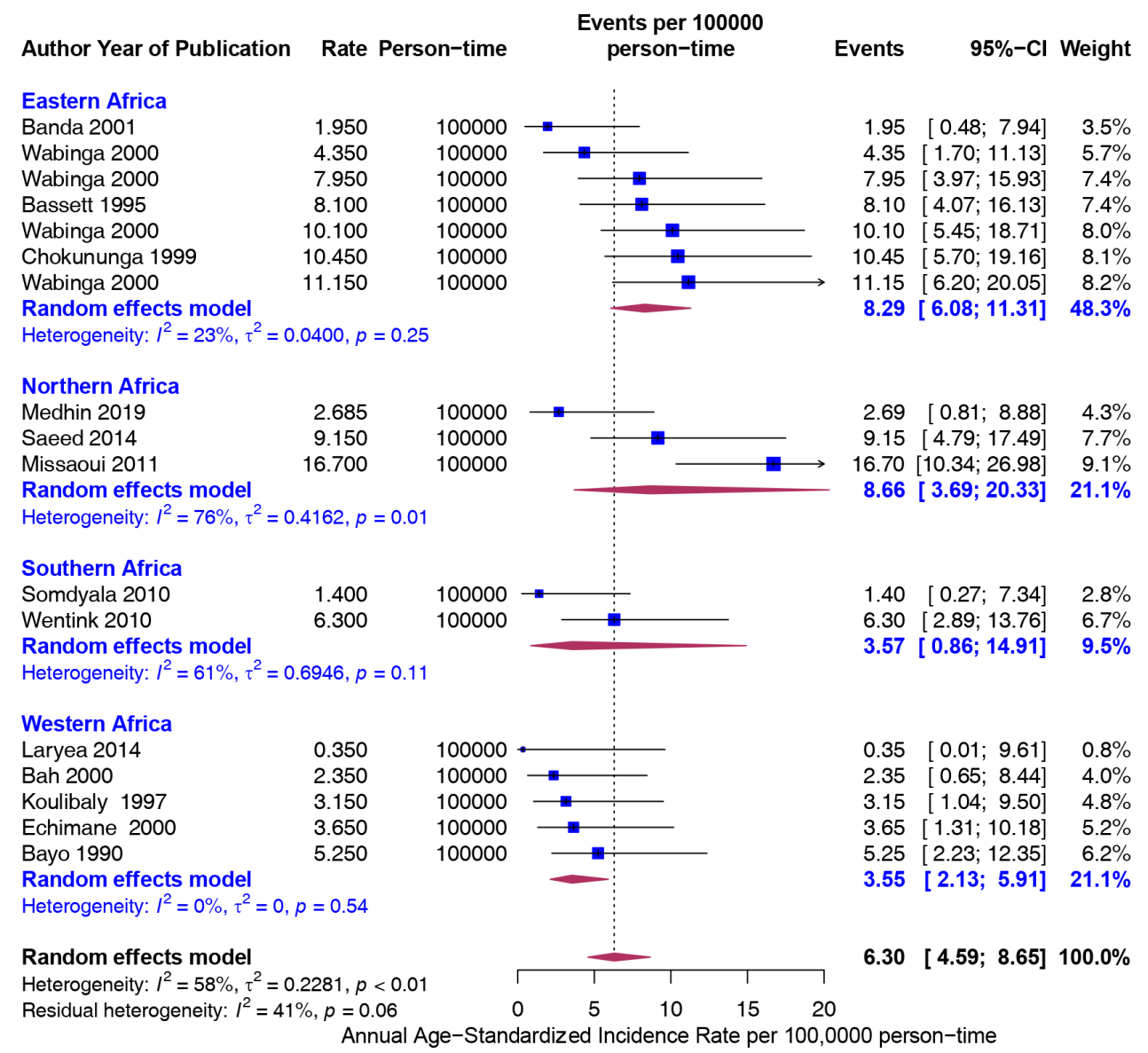

Figure 4 Age-standardised incidence rates (ASIR) (per 100000 population) of colorectal cancer in Africa stratified by United Nations subregions (Northern, Western, eastern and southern Africa). Event values represent the ASIRs of colorectal cancer per 100000 population. Blue squares and their corresponding lines are the point estimates and 95\% Cls. Maroon diamonds represent the pooled estimate of the ASIR, overall and by United Nations subregion (width denotes $95 \% \mathrm{Cl}$ ).

were conference proceedings, 15 came from overlapping populations and 2 were systematic reviews. A total of 14 studies matched all the eligibility criteria. Of the included studies, 2 were from South Africa, ${ }^{15} 162$ from Zimbabwe, ${ }^{17} 18$ and 1 from Ghana, ${ }^{19}$ Eritrea, ${ }^{20}$ The Gambia, ${ }^{21}$ Guinea, ${ }^{22}$ Ivory Coast, ${ }^{23}$ Mali, ${ }^{24}$ Malawi ${ }^{25}$ and Tunisia, ${ }^{26}$ Uganda, ${ }^{27}$ and Sudan $^{28}$ each. The paper by Wabinga $e t$ al from Uganda described cancer incidence at four time points, 1960-1966; 1967-1971; 1991-1994 and $1995-1997 .^{27}$ These time points were analysed independently to allow for trend analysis. A total of 3365 individuals with CRC (mean age, 58 years, $53 \%$ male) were analysed. The percentages of colon and rectal cancer were $65 \%$ and $35 \%$, respectively. The percentage with locally advanced and metastatic disease (stage III and IV) was $57.9 \%$. Adenocarcinoma represented the majority of diagnosed CRCs with a small proportion representing squamous cell carcinoma. Grades 1, 2 and 3 were 32.4 , 60.7 and $8.36 \%$, respectively.

\section{ASIRof CRC per 100000 person-year}

As displayed in figure 2, the overall annual ASIR of CRC per 100000 person-year was 6.30 (95\% CI 4.59 to 8.65). The rates were slightly higher in males than in females but not significantly different (4.76 vs 4.18 ). The heterogeneity was moderate $\left(\mathrm{I}^{2}=58 \%\right)$.

Subgroup analysis was performed by the United Nations regions (North Africa vs SSA) and by United Nations subregions. Although the point estimate was higher in North Africa (8.66) compared with SSA (5.91), the difference was not significant (figure 3 ).

Furthermore, the rates were greater in Eastern (8.29) and Northern (8.66) Africa compared with Western (3.55) and Southern (3.57) Africa, but not significantly different (figure 4). To assess if the rates from recent studies (2000 and later) are higher than older studies (Before 2000), we carried out a stratified analysis. There was no difference in the rates of CRC 5.55 (95\% CI 2.57 to 11.96) and 6.50 (95\% CI 4.72 to 8.94 ), respectively (figure 5 ). The trend in ASIR has remained nearly constant at 4.5 per 100000 population for the last six decades (figure 6).

\section{Study quality, publication bias and sensitivity analyses}

The median study quality score for studies reporting on the incidence was 5 out of 8 (range $=4-9)$. The funnel plot (online supplemental figure 1A) the value of the Egger test $(\mathrm{p}<0.0001)$ and Begg test $(\mathrm{p}<0.0001)$ indicated the presence of publication bias. We used the trim and fill 


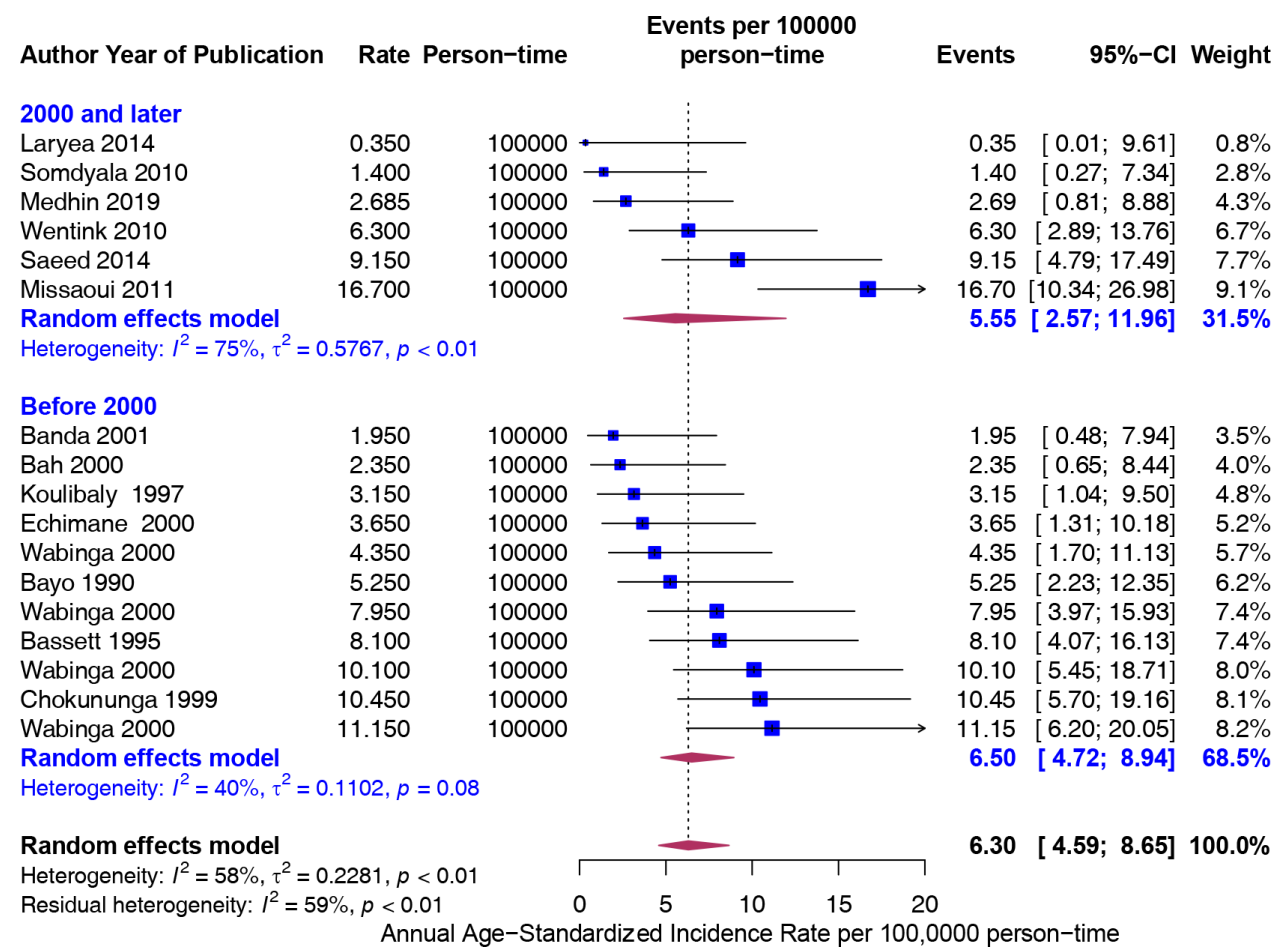

Figure 5 Age-standardised incidence rates (ASIR) (per 100000 population) of colorectal cancer in Africa stratified by year of study (before 2000 and before, 2000 and after). Event values represent the ASIRs of colorectal cancer per 100000 population. Blue squares and their corresponding lines are the point estimates and $95 \% \mathrm{Cls}$. Maroon diamonds represent the pooled estimate of the ASIR, overall and by year categorised as before and after 2000 (width denotes $95 \% \mathrm{Cl}$ ). There is no difference in the rates between the year categories.

method to adjust for the publication bias. If the asymmetry is due to publication bias, the adjusted estimates fall in the range of 5.76 to 12.22 . Finally, Influence sensitivity analyses were by excluding and replacing one study at a

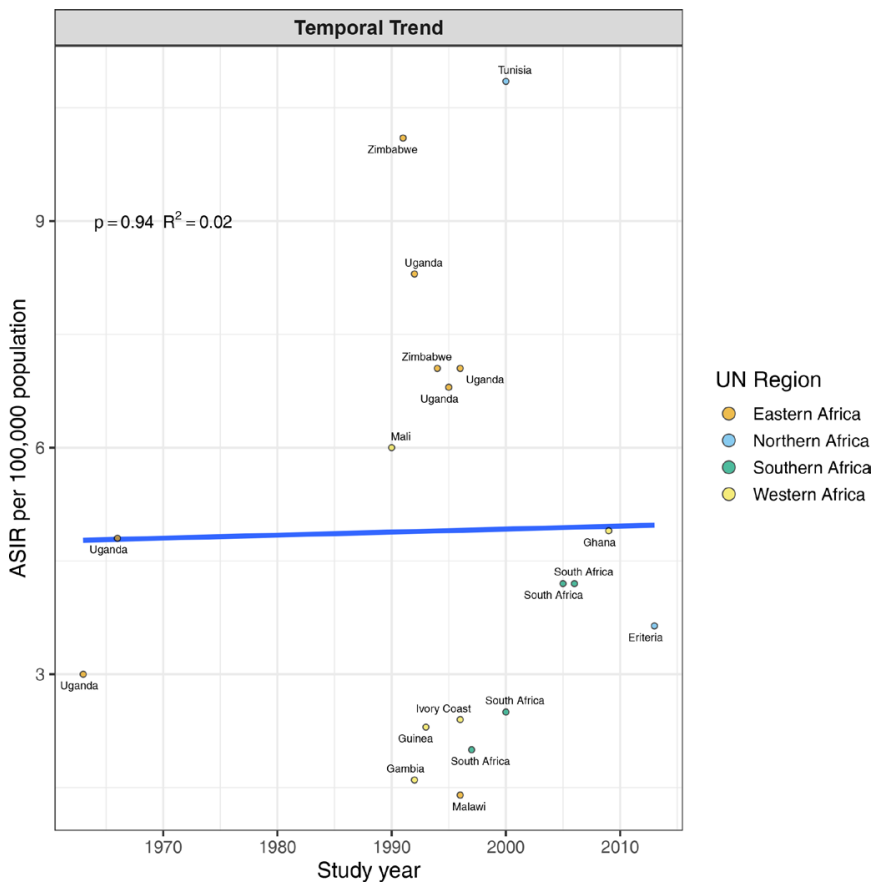

Figure 6 Temporal trends in the incidence rates (per 100000 population) of colorectal cancer in Africa. Rates were constant over time. UN, United Nation. time (leave-one-out method) from the meta-analysis and calculating the pooled ASIR for the remaining studies. No significant change from any of the pooled estimates was observed when other studies were removed in turn. The pooled ASIR ranged from 5.93 to 6.67 (online supplemental figure 1B)

\section{DISCUSSION}

This paper provides a comprehensive meta-analysis of the patterns and trends in the CRC incidence in Africa. The estimated annual ASIR of CRC per 100000 persons was 6.30. This rate is higher than reported in a 2012 systematic review of only SSA countries by Graham et al which reported a crude incidence rate of 4.04 per $100000 .^{5}$ When compared with SSA, North Africa had the highest ASIR of 8.66, while SSA had an ASIR of 5.91. Middle Africa was not represented in this meta-analysis.

CRC is known to be the most common malignancy of the GI tract, ${ }^{29}$ and while previously thought to be a rare malignancy in Africa, recent data is proving otherwise. ${ }^{30}{ }^{31}$ In addition to being more common than previously recognised, CRC in Africa tends to present more commonly in young adults. ${ }^{31}$ This trend in young adults is similar to current trends in the USA, Asia and Europe, where patients usually present with advanced stage, leftsided tumours and poor histology. ${ }^{32}$

In the USA, CRC is the third most common cancer in both men and women but the second-leading cause 
of cancer death. ${ }^{33}$ Rates of CRC in the USA have been declining since the mid-1980s in patients older than 50 years old, mainly due to increased cancer screening and changes in lifestyle. This is in sharp contrast to young adults $<50$ years old where there is an expected exponential increase between 2020 and 2030. ${ }^{34}$ According to a study by Siegel $e t$ al, the ASIR of CRC in the USA from 2012 to 2016 was 38.7 per 100000 persons. ${ }^{21}$ Furthermore, in the USA, the ASIR in Blacks from 2012 to 2016 was 45.7 compared with 38.6 in nonHispanic whites. ${ }^{22}{ }^{23}$ People of African descent present at a younger age and with more advanced disease at diagnosis and have the highest mortality rate among different ethnic groups. ${ }^{35}$ Factors responsible for these differences are multifactorial, including known health disparities, socioeconomic status, genetic factors and dietary influences. ${ }^{36} 37$

Even though our study provides much lower ASIRs, it is assumed that these do not accurately reflect the actual incidence of CRC in Africa. We suspect this number to be much higher. According to the study by Laiyemo et al, there is no population-based CRC screening or guidelines in any African country to date. ${ }^{38}{ }^{39}$ To better understand the true incidence rates of CRC in Africa, standardised screening guidelines must be established. Given the lack of screening, patients commonly present with advanced disease. More countries are implementing and establishing PBCR $^{39}$ described in this study by Omonisi et al. These registries should inform us of more specific country incidence rates and allow for further population-based studies that could unravel the mysteries behind the increased risk of CRC in people of African descent.

The present analysis has major strengths. First, all United Nations regions (North Africa, SSA) and subregions (Eastern, Western, Southern and Northern Africa) of Africa were represented (except Middle Africa). Thus, our findings can be generalisable at the regional level of Africa. Second, we included recent estimates of CRC in Africa. The present estimates are the most updated figures of the rates of colorectal cancer in Africa and thus can be used to inform the prevention and control strategies. Nevertheless, this study has some limitations. First, country-level data came from only $18 \%$ of the continent, meaning most countries were not represented due to the lack of published literature on CRC incidence in these countries. Therefore, the estimates may not be generalisable at the country level. To mitigate this limitation, we conducted subgroup analysis by African regions (North Africa, SSA) and subregions (Eastern, Western, Southern and Northern Africa) to explore possible regional and subregional specific rates. Second, the estimates could suffer from potential selection bias due to a lack of random population-based studies such as those conducted by the demographic and health surveys programme and countrybased cancer registries. However, the present systematic review and meta-analysis provides the updated estimates of CRC in Africa using the best available information, and we have applied rigorous sensitivity analysis to minimise bias.

\section{CONCLUSION}

CRC estimates in Africa are heterogeneous and could be underestimated. Population-based CRC data are scarce in Africa. High-quality data collection systems such as PBCR may facilitate country-specific rates and provide accurate information which would be lucrative to the consideration of resources needed for screening, early detection, treatment and improving overall patient outcomes.

\section{Author affiliations}

${ }^{1}$ Department of Medicine, Division of Hematology/Oncology, Vanderbilt University Medical Center, Nashville, Tennessee, USA

${ }^{2}$ Department of Public Health Sciences, Penn State Health Milton S Hershey Medical Center, Hershey, Pennsylvania, USA

${ }^{3}$ Department of Surgery, Penn State Health Milton S Hershey Medical Center, Hershey, Pennsylvania, USA

Contributors NA, CE and PS conceived and designed the study. NA, MT and PS extracted data. PS performed statistical analysis, created figures and is the guarantor of the overall content. NA and PS drafted the manuscript and made subsequent revisions. CE and J0 supervised the study. EJO, CP and CS critically revised the manuscript for intellectual content. All authors read and approved the final version of this manuscript.

Funding The authors have not declared a specific grant for this research from any funding agency in the public, commercial or not-for-profit sectors.

Competing interests None declared.

Patient consent for publication Not applicable.

Ethics approval This study does not involve human participants.

Provenance and peer review Not commissioned; externally peer reviewed.

Data availability statement All data relevant to the study are included in the article or uploaded as online supplemental information. All data needed to reproduce the results are included in the manuscript.

Supplemental material This content has been supplied by the author(s). It has not been vetted by BMJ Publishing Group Limited (BMJ) and may not have been peer-reviewed. Any opinions or recommendations discussed are solely those of the author(s) and are not endorsed by BMJ. BMJ disclaims all liability and responsibility arising from any reliance placed on the content. Where the content includes any translated material, BMJ does not warrant the accuracy and reliability of the translations (including but not limited to local regulations, clinical guidelines, terminology, drug names and drug dosages), and is not responsible for any error and/or omissions arising from translation and adaptation or otherwise.

Open access This is an open access article distributed in accordance with the Creative Commons Attribution Non Commercial (CC BY-NC 4.0) license, which permits others to distribute, remix, adapt, build upon this work non-commercially, and license their derivative works on different terms, provided the original work is properly cited, appropriate credit is given, any changes made indicated, and the use is non-commercial. See: http://creativecommons.org/licenses/by-nc/4.0/.

\section{ORCID iDs}

Paddy Ssentongo http://orcid.org/0000-0003-1565-5731

Colette Pameijer http://orcid.org/0000-0002-6204-8654

\section{REFERENCES}

1 Bray F, Ferlay J, Soerjomataram I, et al. Global cancer statistics 2018: GLOBOCAN estimates of incidence and mortality worldwide for 36 cancers in 185 countries. CA Cancer J Clin 2018;68:394-424.

2 Arnold M, Sierra MS, Laversanne M, et al. Global patterns and trends in colorectal cancer incidence and mortality. Gut 2017;66:683-91. 
3 Negin J, Cumming R, de Ramirez SS, et al. Risk factors for noncommunicable diseases among older adults in rural Africa. Trop Med Int Health 2011;16:640-6.

4 Anoba IB. How a population of 4.2 billion could impact Africa by 2100: the possible economic. The SAIS Review of International Affairs, 2019.

5 Graham A, Adeloye D, Grant L, Davies Adeloye LG, Theodoratou E, et al. Estimating the incidence of colorectal cancer in sub-Saharan Africa: a systematic analysis. J Glob Health 2012;2:020404.

6 Parkin DM, Bray F, Ferlay J, et al. Cancer in Africa 2012. Cancer Epidemiol Biomarkers Prev 2014;23:953-66.

7 Stroup DF, Berlin JA, Morton SC, et al. Meta-Analysis of observational studies in epidemiology: a proposal for reporting. Meta-analysis of observational studies in epidemiology (moose) group. JAMA 2000;283:2008-12.

8 Moher D, Liberati A, Tetzlaff $\mathrm{J}$, et al. Preferred reporting items for systematic reviews and meta-analyses: the PRISMA statement. PLOS Med 2009;6:e1000097.

9 Peterson J, Welch V, Losos M. The Newcastle-Ottawa scale (NOS) for assessing the quality of nonrandomised studies in meta-analyses. Ottawa: Ottawa Hospital Research Institute, 2011.

10 Parkin D. Comparability and quality control in cancer registration. IARC Technical report. . IARC Publications, 1994: 19. 18-19.

11 Schwarzer G, Carpenter JR, Rücker G. Meta-Analysis with R. Springer, 2015.

12 DerSimonian R, Kacker R. Random-effects model for meta-analysis of clinical trials: an update. Contemp Clin Trials 2007;28:105-14.

13 Higgins JPT, Thompson SG, Deeks JJ, et al. Measuring inconsistency in meta-analyses. BMJ 2003;327:557-60.

14 Egger M, Davey Smith G, Schneider M, et al. Bias in meta-analysis detected by a simple, graphical test. BMJ 1997;315:629-34.

15 Wentink MQ, Räkers M, Stupart DA, et al. Incidence and histological features of colorectal cancer in the Northern Cape Province, South Africa. S Afr J Surg 2010;48:109-13.

16 Somdyala NI, Bradshaw D, Gelderblom WC, et al. Cancer incidence in a rural population of South Africa, 1998-2002. Int J Cancer 2010;127:2420-9.

17 Chokunonga E, Levy LM, Bassett MT, et al. Cancer incidence in the African population of Harare, Zimbabwe: second results from the cancer registry 1993-1995. Int J Cancer 2000;85:54-9.

18 Bassett MT, Chokunonga E, Mauchaza B, et al. Cancer in the African population of Harare, Zimbabwe, 1990-1992. Int J Cancer 1995;63:29-36.

19 Laryea DO, Awuah B, Amoako YA, et al. Cancer incidence in Ghana, 2012: evidence from a population-based cancer registry. BMC Cancer 2014:14:362.

20 Medhin LB, Achila OO, Abrham AT, et al. Incidence of colorectal cancer in Eritrea: data from the National health laboratory, 20112017. PLoS One 2019;14:e0224045.

21 Siegel RL, Miller KD, Goding Sauer A, et al. Colorectal cancer statistics, 2020. CA Cancer J Clin 2020;70:145-64.
22 Koulibaly M, Kabba IS, Cissé A, et al. Cancer incidence in Conakry, guinea: first results from the cancer registry 1992-1995. Int J Cancer 1997;70:39-45.

23 Echimane AK, Ahnoux AA, Adoubi l, et al. Cancer incidence in Abidjan, Ivory Coast: first results from the cancer registry, 1995-1997. Cancer 2000;89:653-63.

24 Bayo S, Parkin DM, Koumaré AK, et al. Cancer in Mali, 1987-1988. Int J Cancer 1990;45:679-84.

25 Banda LT, Parkin DM, Dzamalala CP, et al. Cancer incidence in Blantyre, Malawi 1994-1998. Trop Med Int Health 2001;6:296-304.

26 Missaoui N, Jaidaine L, Abdelkader AB, et al. Colorectal cancer in central Tunisia: increasing incidence trends over a 15 -year period. Asian Pac J Cancer Prev 2011;12:1073-6.

27 Wabinga HR, Parkin DM, Wabwire-Mangen F, et al. Trends in cancer incidence in Kyadondo County, Uganda, 1960-1997. Br J Cancer 2000;82:1585-92.

28 Saeed IE, Weng H-Y, Mohamed KH, et al. Cancer incidence in Khartoum, Sudan: first results from the cancer registry, 2009-2010. Cancer Med 2014;3:1075-84.

29 Siegel RL, Miller KD, Jemal A. Cancer statistics, 2015. CA Cancer J Clin 2015;65:5-29.

30 Agyemang-Yeboah F, Yorke J, Obirikorang C, et al. Patterns and presentations of colorectal cancer at Komfo-Anokye teaching hospital Kumasi, Ghana. Pan Afr Med J 2017;28:142.

31 Irabor D, Adedeji OA. Colorectal cancer in Nigeria: 40 years on. A review. Eur J Cancer Care 2009;18:110-5.

32 Mauri G, Sartore-Bianchi A, Russo A-G, et al. Early-Onset colorectal cancer in young individuals. Mol Oncol 2019;13:109-31.

33 Gu M, Thapa S. Colorectal cancer in the United States and a review of its heterogeneity among Asian American subgroups. Asia Pac J Clin Oncol 2020;16:193-200.

34 Bailey CE, Hu C-Y, You YN, et al. Increasing disparities in the agerelated incidences of colon and rectal cancers in the United States, 1975-2010. JAMA Surg 2015;150:17-22.

35 Jackson CS, Oman M, Patel AM, et al. Health disparities in colorectal cancer among racial and ethnic minorities in the United States. $J$ Gastrointest Oncol 2016;7:S32.

$36 \mathrm{Ou}$ J, Carbonero F, Zoetendal EG, et al. Diet, microbiota, and microbial metabolites in colon cancer risk in rural Africans and African Americans. Am J Clin Nutr 2013;98:111-20.

37 Sharma I, Kim S, Sridhar S, et al. Colorectal cancer: an emphasis on factors influencing racial/ethnic disparities. Crit Rev Oncog 2020;25:151-60.

38 Laiyemo AO, Brawley O, Irabor D, et al. Toward colorectal cancer control in Africa. Int J Cancer 2016;138:1033-4.

39 Omonisi AE, Liu B, Parkin DM. Population-Based cancer registration in sub-Saharan Africa: its role in research and cancer control. JCO Glob Oncol 2020;6:1721-8. 